\title{
Amoeboid swimming in compliant channel
}

\author{
Swapnil Dalal, ${ }^{a}$ Alexander Farutin, ${ }^{a}$ and Chaouqi Misbah ${ }^{* a}$ \\ ${ }^{a}$ Laboratoire Interdisciplinaire de Physique, Université Grenoble \\ Alpes and CNRS, F-38000 Grenoble, France
}

*E-mail: chaouqi.misbah@univ-grenoble-alpes.fr 


\begin{abstract}
Several prokaryotes and eukaryotic cells swim in presence of deformable and rigid surfaces that form confinement. Most commonly observed examples from biological systems are motility of leukocytes and pathogens present within blood suspension through microvascular network, locomotion of eukaryotic cells such as immune system cells, cancerous cells through interstices between soft interstitial cells and extracellular matrix within interstitial tissue. This motivated us to investigate numerically flow dynamics of amoeboid swimming in flexible channel. The effects of wall stiffness and channel confinement on the flow dynamics and swimmer motion are studied. The swimmer motion through the flexible channel is substantially decelerated compared to the rigid channel. The strong confinement in amply flexible channel imprisons the swimmer by severely restricting its forward motion. The swimmer velocity in a stiff channel displays nonmonotonic variation with the confinement while it shows monotonic reduction in a highly flexible channel. The physical rationale behind such distinct velocity behaviour in flexible and rigid channels is illustrated using instantaneous flow field and flow history displayed by the swimmer. This behavior follows from a subtle interplay between the shape changes exhibited by the swimmer and the wall compliance. This study may aid in understanding influence of elasticity of surrounding environment on cell motility in immunological surveillance and invasiveness of cancer cells.
\end{abstract}




\section{Introduction}

Last two decades have witnessed plethora of studies from various interdisciplinary research groups who investigated different aspects of hydrodynamics of microorganism swimming due to their significance in aquatic ecosystem $[1,2,3]$, proliferation of pathogenic infections $[4,5]$, cellular scale hydrodynamics $[6,7,8]$ and designing of efficient swimming microrobots [9, 10, 11]. These microswimmers are often surrounded by active or passive particles such as microbes and cells, rigid or deforming surfaces such as microvascular network, cell walls, interstitium, microtubules, membranes. Pathogens exploit these surroundings to facilitate their motility. The hydrodynamic interaction between microswimmers and neighbouring surfaces influences swimmer speed, its efficiency, trajectory, orientation and collective behaviour.

Microswimmers exhibit peculiar behaviour in presence of surfaces. Some bacteria such as Escherichia Coli exhibit circular clockwise motion in presence of solid surface $[12,13,14]$, anticlockwise circular trajectory near liquid-air free surface $[15,16,14]$ and reversal of its direction in presence of obstacle [17]. Recent experimental $[18,19]$ and theoretical [20] studies have reported changes in the direction of circular trajectory of $\mathrm{E}$. Coli in presence of polymers in the fluid near solid wall and surfactant in the solution near free surface. Crowdy et al. [21] showed that free capillary surface can be deformed to induce steady locomotion parallel to the free interface. The bacteria tends to be trapped close to the surface and forms aggregates $[22,23,24,25]$. This microbial behaviour is purely governed by passive hydrodynamic interaction $[12,15]$. This shows that surface plays pivotal role in controlling hydrodynamic behaviour of microswimmers. Thus new strategies can be developed by tuning hydrodynamic properties of surface and direct motility and sorting of microswimmers purely based on their hydrodynamic interaction with the surface and eliminate usage of external means such as pumping, valving, chemical gradient etc, to direct their motion.

Recent studies $[26,27,28,29,30]$ have reported that several eukaryotic cells such as neutrophils, T-lymphocytes and microorganisms such as Dictyostelium amoeba can exhibit shape deformation based pure swimming away from the substrate instead of conventional adhesion assisted crawling on the substrate and display comparable swimming speed (as fast as crawling). This corroborates the observed fact that leukocytes can move in extracellular matrix despite the inactivity of the principal adhesion molecules (integrins) [29]. These findings motivated studies on locomotion of eukaryotic cells and microorganisms such as Euglena [31], Eutreptiella Gymnastica [32] by considering deformation based pure swimming and eliminate complexity involved in adhesion assisted crawling. The swimming dynamics of such deformable non-adherent self propelled particles has been investigated by several numerical and experimental studies $[33,34,35,36,37,31,38,39,40,41,42,43]$.

Many biological systems involve motility of microorganisms, pathogens and eukaryotic cells through confinement formed by deformable surfaces within interstitium and microvascular network $[44,45,8,46,47,48]$. The stiffness of 
extracellular matrix (ECM) is known to control cell locomotion $[49,50,51]$. Some cancerous cells enhance stiffness of surrounding ECM environment to facilitate their motility and invasiveness. For example, malignant tumor cells such as astrocytoma, breast cancer, pancreatic adenocarcinoma remodel and stiffen surrounding extracellular matrix in order to enhance cell motility and proliferation $[52,53,54,55,56,57]$. The cell locomotion through ECM is governed by cell type, motility strategy employed by the cell (such as adhesion assisted crawling, pure swimming), composition and elasticity of surrounding ECM etc. In order to decipher such complex hydrodynamics question, it seems logical to simplify the problem by ignoring the role of adhesion and focusing on the effects of surface deformability on pure swimming based cell locomotion. The numerical investigation of Ledesma-Aguilar and Yeomans [58] reported that motility of dipolar swimmer is enhanced due to elastic confinement while Fauci and McDonald [59] and Shaik et al. [60] for spermatozoid and squirmer model respectively, observed that the swimmer velocity reduces with an increase in the wall deformability. This points to the fact that the nature of the swimmer may play a decisive role.

In this article, we numerically investigate the effects of the wall flexibility and the channel confinement on the migration, speed, trajectory and efficiency of an amoeboid swimmer in two dimension. The article is organized as follow. Section 2 presents governing equations, models for passive, active and elastic forces and numerical methodology. The results are discussed in section 3. The swimming mechanism of amoeboid swimmer in rigid and flexible channels is explained in section 3.3. Concluding remarks and future lines of research are the subject of section 4 .

\section{Formulation}

The deformable microswimmer with an inextensible membrane (say a phospholipid membrane) denoted by $\Omega_{3}$ that encapsulates Newtonian fluid is suspended in a Newtonian matrix delimited by the two flexible walls, $\Omega_{1}$ and $\Omega_{2}$, that forms flexible channel of an undeformed width $W$ as shown in the schematic of two dimensional system in figure 1. For simplicity, the viscosity, $\eta$, of the enclosed fluid is taken to be constant and is equal to that of the suspending fluid. The reasoning behind this assumption is discussed in the conclusion section.

We define the effective radius of the swimmer as $R_{o}=\sqrt{A_{o} / \pi}$, where $A_{o}$ is the enclosed area and $P_{o}$ is the perimeter (which is conserved due to membrane inextensibility). The excess perimeter, $\Gamma=P_{o} /\left(2 \pi R_{o}\right)-1$, describes the degree of deflation of swimmer shape. $\Gamma=0$ corresponds to a circular shape while larger $\Gamma$ represents shape deformation and deviation from a circular shape. We model shape deformation based propulsion of a swimmer referred to as amoeboid swimming. The swimmer's internal propulsion machinery responsible for its shape changes and motion are modeled by assuming that the swimmer deploys active force distribution along its membrane. The active force distribution depends on time and on material point on the membrane. 


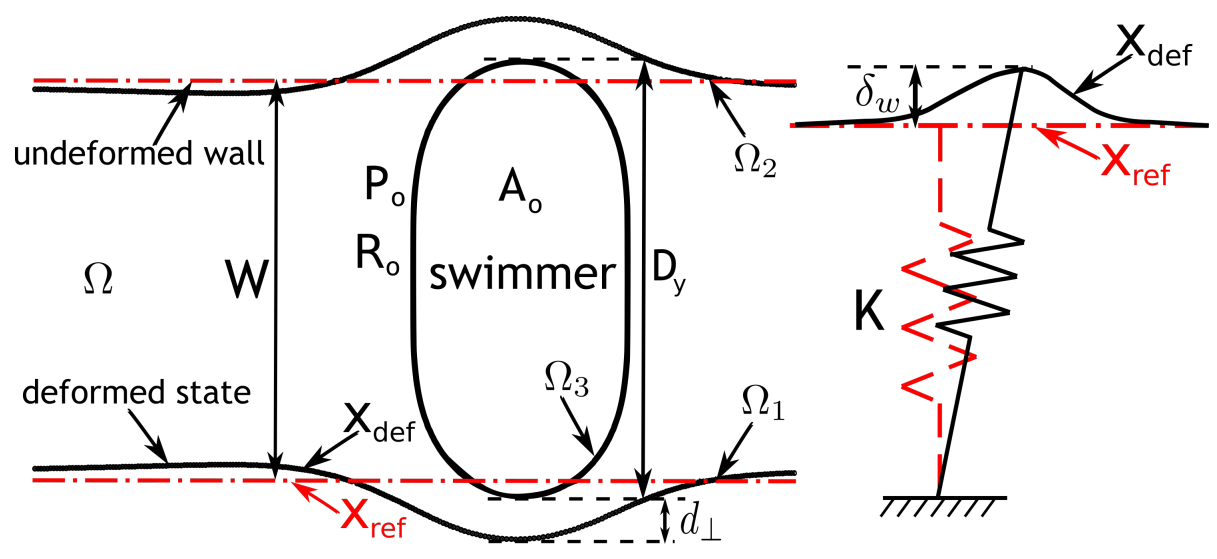

Figure 1: (color online) Schematics of amoeboid swimmer suspended in flexible channel. Swimmer deforms elastic wall from its reference configuration $\mathbf{x}_{r e f}$ to the deformed configuration $\mathbf{x}_{d e f}$. Linear elastic law governs the wall deformation.

The flow of microswimmer in an incompressible viscous Newtonian fluid bounded by the flexible walls (such flow is characterized by very small Re $10^{-2}-10^{-3}$ due to both small swimming velocity and small swimmer size) is governed by continuity and Stokes equation.

$$
\begin{array}{r}
\nabla \cdot \mathbf{u}=0, \\
-\nabla p+\eta \nabla^{2} \mathbf{u}+\mathbf{f}=0,
\end{array}
$$

where $\mathbf{u}$ is fluid velocity, $p$ is pressure and $\mathbf{f}$ is the total force acting along the membrane of the swimmer. The major contribution to this total force is the active force distribution $\left(\mathbf{f}_{a}\right)$ imposed on the swimmer. In order to replicate cell locomotion, the swimmer membrane is modeled as a two dimensional incompressible fluid. This conditions is fulfilled by local membrane inextensibility which induces tension like internal force within the swimmer membrane that resists tendency of the active force to extend or compress the membrane. The changes in the membrane curvature with time induces bending force on the membrane. The tension and bending forces acting on the membrane are referred as passive force contribution $\left(\mathbf{f}_{p}\right)$. The flexible wall is modeled as an extensible membrane and it comprises of restoring elastic force contribution $\left(\mathbf{f}_{w}\right)$ that arises due to deviation of the membrane position from its planar reference configuration. We now discuss the framework used for modeling of passive, active and elastic forces acting on the membrane.

\subsection{Passive membrane forces}

The swimmers surface is modeled as an incompressible and inextensible membrane. The inextensibility of the swimmer membrane is conserved locally by 
employing local Lagrange multiplier $\zeta(s, t)$. Using Helfrich model [61, 62], the elastic energy of the membrane can be expressed as composition of bending energy and tension.

$$
E=\frac{\kappa_{b}}{2} \oint_{\Omega_{i}} H^{2} d s+\oint_{\Omega_{i}} \zeta(s, t) d s
$$

where $H$ is the local membrane curvature. $s$ is the arc length and $\kappa_{b}$ is the bending energy modulus. The force acting on the membrane is obtained by functional derivative of the membrane energy, $E$, with respect to the membrane displacement as shown in Kaoui et al. [62].

$$
\mathbf{f}_{p}=\kappa_{b}\left(\frac{\partial^{2} H}{\partial s^{2}}+\frac{H^{3}}{2}\right) \mathbf{n}-H \zeta \mathbf{n}+\frac{\partial \zeta}{\partial s} \mathbf{t}
$$

where $\mathbf{n}$ and $\mathbf{t}$ are local normal and tangent vector respectively.

\subsection{Active force model}

Several microorganisms such as Eutreptiella Gymnastica [32] and eukaryotic cells such as neutrophiles, fibroblasts, leukocytes, cancerous cells undergo shape deformation in order to propel themselves. We represent active force distribution as time dependent and material point position function [35, 36, 37]. The active force on the swimmer membrane is decomposed into Fourier series as,

$$
\mathbf{f}_{a}(\alpha, t)=\sum_{k=-k_{\max }, k \mid \neq 0,1}^{k_{\max }} \hat{F}_{k}(t) e^{i k \alpha} \cdot \mathbf{n} .
$$

Here $\alpha$ is a normalized arc length, $\alpha=2 \pi s / P_{o}$. We consider that the active force acts along normal direction. In appendix, we have shown that it is possible to redefine the tension force (Lagrange multiplier $(\zeta)$ ) and express active force in terms of normal component only. The isolated nature of the swimmer imposes that the total force and torque exerted by the swimmer on the fluid vanishes at every instant.

$$
\oint_{\Omega_{3}} \mathbf{f} d s=0, \quad \oint_{\Omega_{3}} \mathbf{r} \times \mathbf{f} d s=0
$$

These two vectorial conditions yields three scalar equations which are linear in terms of active force. The Fourier coefficients of the system depend on the swimmer shape, which is unknown a priori and equation (5) imposes linear relations between Fourier coefficients $\hat{F}_{k}$. These relations could not reduce number of independent Fourier coefficients as resulting system of equations was sometimes ill-conditioned. The additional term, $\mathbf{f}_{0}+\widetilde{f}_{0} \mathbf{t}$, is added to the active force whose first component is constant while second component is purely tangential. The components, $\mathbf{f}_{0}$ and $\widetilde{f}_{0}$, can be expressed in terms of $\hat{F}_{k}$ using equation (5). The resulting system of linear equations, comprising of $\mathbf{f}_{0}$ whose coefficients are 
proportional to the swimmer perimeter and $\widetilde{f}_{0}$ whose coefficients are proportional to the swimmer area, becomes nonsingular irrespective of the swimmer evolution as swimmer perimeter and area are positive definite quantities (see [37]). Thus, the total force acting on the swimmer membrane is,

$$
\mathbf{f}=\mathbf{f}_{p}+\mathbf{f}_{a}+\mathbf{f}_{0}+\widetilde{f}_{0} \mathbf{t} .
$$

For simplicity, we restrict Fourier series expansion of the active force in equation (4) to $k_{\max }=3$. In earlier studies [35, 36, 37], we have observed that including higher harmonics for the active force $\left(\mathbf{f}_{a}\right)$ representation does not alter the results. Due to fluid incompressibility, the mode $k=0$ does not contribute. For simplicity, we set $F_{1}=0$. In addition, we impose mirror symmetry of the swimmer, so that active force is written as,

$$
\mathbf{f}_{a}(\alpha, t)=\left(2 F_{2}(t) \cos (2 \alpha)+2 F_{3}(t) \cos (3 \alpha)\right) \mathbf{n} .
$$

We express quantities $F_{2}$ and $F_{3}$ as simple time dependent function that induces non reciprocal cyclic forward motion of the swimmer and overcome constraints of the Scallop theorem.

$$
F_{2}(t)=-A_{2} \cos (\omega t), \quad F_{3}(t)=A_{3} \sin (\omega t) .
$$

For simplicity, we set $A_{2}=A_{3}=A$. Thus the active force consists of four parameters $A$, scalar $\widetilde{f}_{0}$ and two components of $\mathbf{f}_{0}$. The force-free and torquefree conditions (equation (5)) are used to deduce $f_{0 x}, f_{0 y}$ and $\widetilde{f}_{0}$ as a function of force amplitude, $A$. Thus the active forces can be controlled by adjusting single parameter which is the force amplitude, $A$.

\subsection{Modeling of flexible wall}

We adopted simple plate spring type compliant wall model $[63,64,65,66,67]$ for modeling flexible walls as shown in schematics in figure 1 . The undeformed reference configuration of the flexible wall is $\mathbf{x}_{r e f}(\mathbf{x})$. The presence of swimmer disturbs the flow field in the vicinity of elastic wall resulting in its deformation. The deformed configuration of the flexible wall is $\mathbf{x}_{\text {def }}(\mathbf{x}, t)$. In the absence of flow disturbance, deformed wall configuration $\mathbf{x}_{\text {def }}(\mathbf{x}, t)$ coincides with its reference configuration $\mathbf{x}_{r e f}(\mathbf{x})$ and does not induce elastic force. The flow disturbance displaces flexible wall to the deformed configuration resulting in an elastic force acting on each material point of the flexible wall. These elastic forces are modeled using linear elastic law [68] given by,

$$
\mathbf{f}_{w}(\mathbf{x}, t)=-K\left[\mathbf{x}_{d e f}(\mathbf{x}, t)-\mathbf{x}_{r e f}(\mathbf{x})\right] .
$$

Here $K$ is spring stiffness. Large spring stiffness represents rigid wall $(K \rightarrow \infty)$ while small spring stiffness depicts soft wall $(K \rightarrow 0)$. This spring stiffness is related to the Young's modulus [68] and will be discussed in the section 2.5. We introduce a small bending contribution to the force in order to suppress numerical instabilities and sharp deformation of the wall (such as cusps). This 
bending term is merely introduced as a numerical regularization, rather than as a physical effect. The non-slip boundary condition is imposed on the flexible wall surface. The periodic boundary condition is employed along the channel axis. The channel length is chosen large enough to approximate the case of a single swimmer in an infinite channel. The space between flexible walls and top or bottom boundaries of a computational domain is chosen large enough to eliminate hydrodynamic effects arising due to presence of finite space on either sides of the flexible walls.

\subsection{Boundary integral method}

The linearity of Stokes equation allows velocity field in a fluid domain to be represented using boundary integral equation as a superposition of contribution from $i$ ) the imposed flow, $i i$ ) the membrane forces at the interface and $i$ ii) contribution due to viscosity contrast between ambient fluid and internal fluid $[69,70]$. The contribution due to first component is neglected due to absence of the imposed flow. As viscosity of ambient and internal fluid is equal, contribution due to third component vanishes. The velocity at some point $\mathbf{r}_{o}$ in a fluid domain is given by,

$$
\mathbf{u}\left(\mathbf{r}_{o}\right)=\frac{1}{4 \pi \eta_{o}} \sum_{i} \oint_{\Omega_{i}} d s\left(\mathbf{r}_{m}\right) \mathbf{f}\left(\mathbf{r}_{m}\right) \mathbf{G}\left(\mathbf{r}_{o}, \mathbf{r}_{m}\right),
$$

where integrals are performed around membrane contour, $\Omega_{i}$, of the swimmer $\left(\Omega_{3}\right)$ and the deformable walls $\left(\Omega_{1}, \Omega_{2}\right), \mathbf{G}\left(\mathbf{r}_{o}, \mathbf{r}_{m}\right)$ is an appropriate Green's function of the Stokes flow for the velocity that satisfies boundary conditions at the system boundaries (it vanishes at the wall and infinity), $\mathbf{r}_{o}$ and $\mathbf{r}_{m}$ are target and source points, $\mathbf{f}\left(\mathbf{r}_{m}\right)=\mathbf{f}_{p}+\mathbf{f}_{a}+\mathbf{f}_{w}$ is hydrodynamic traction jump across the membrane and comprises of contribution due to passive (equation (3)), active (equation (4)) and wall elastic forces (equation (8)) and $\mathbf{n}\left(\mathbf{r}_{m}\right)$ is outward unit normal vector at the membrane. The term on right side of the equation (9) is referred as single layer potential integral. Boundary integral method (BIM) involves discretization of the membrane and the wall surfaces. All quantities are discretized using Fourier spectral boundary integral method in space [71].

\subsubsection{Spectral boundary integral method}

We use Fourier basis discretization of all functions and compute all derivatives in Fourier domain in order to preserve high accuracy [71]. Fourier basis is used to represent membrane interface. The interface $(\mathbf{x}(\alpha))$ in a parametrized domain is divided into $M$ uniformly spaced positions $\left\{\alpha_{k}=2 \pi(k-1) / M\right\}_{k=1}^{M}$. The

interface positions and its derivatives are represented in terms of Fourier basis as,

$$
\mathbf{x}(\alpha)=\sum_{k=-M / 2}^{M / 2} \hat{\mathbf{x}}(k) e^{i k \alpha}, \quad \mathbf{x}_{\alpha}=\sum_{k=-M / 2}^{M / 2}(-i k) \hat{\mathbf{x}}(k) e^{i k \alpha}, \quad \alpha \in[0,2 \pi] .
$$


where the subscript $\alpha$ refers to derivative with respect to $\alpha$. FFT is used to evaluate discrete Fourier transform of $\mathbf{x}(\alpha)$ and inverse Fourier transform of $\hat{\mathbf{x}}(k)$. The parametrized arc length is represented by $s(\alpha)=\int_{0}^{\alpha}\left|\mathbf{x}_{\alpha}\right| d \alpha$. The derivative of any function $Y$ with respect to arc length is evaluated using FFT as,

$$
Y_{s}=\frac{\partial Y}{\partial s}=\frac{Y_{\alpha}}{s_{\alpha}}=\frac{\partial \alpha}{\partial s} \frac{\partial Y}{\partial \alpha}=\frac{1}{|\partial \mathbf{x} / d \alpha|} \frac{\partial Y}{d \alpha}
$$

The integrand for single layer potential in equation (9) exhibits singular behaviour as Green kernel, $\mathbf{G}$, diverges in the vicinity of the pole where target $\left(\mathbf{r}_{o}\right)$ and source points $\left(\mathbf{r}_{m}\right)$ coincide. This singular behaviour is circumvented by using singularity subtraction method (SS) discussed in Farutin et al. [72]. The regularized smooth integrals are evaluated using trapezoidal rule. The details of this approach are discussed in Farutin et al. [72]. The membrane positions are advected by integrating in time using explicit Euler scheme as, $\mathbf{x}_{k}(t+d t)=\mathbf{x}_{k}(t)+\mathbf{u}_{k}(t) d t$.

\subsection{Nondimensional parameters}

Besides the excess perimeter, $\Gamma$, defined earlier, there are other dimensionless parameters that are used to analyse flow behaviour. The confinement strength of undeformed flexible channel is defined as $C=2 R_{o} / W$, where $W$ is the channel width of the undeformed channel. The time period of swimming stroke is given by $T_{s}=2 \pi / \omega$ and $A$ is the amplitude of active force distribution. The important dimensionless parameters explored in this study are,

- the confinement strength, $C=2 R_{o} / W$,

- the dimensionless wall stiffness, $K_{w}=K R_{o} / A$ and

- the dimensionless active force, $S=A /(\omega \eta)$.

The wall stiffness, $K$, is, as an order of magnitude, of order Young's modulus, $E$, so that $K_{w} \sim E / A$, where $A$ is force per unit area. The swimming microorganisms such as green algae Chlamydomonas reinhardtii of size $\sim 5 \mu \mathrm{m}$ deploys propulsive forces in the range of $\sim 1-10 p N[73,74,75]$. Bacteria, such as E. coli, generates thrust force of order $\sim 0.4-1 p N[76,22,77]$. The elastic moduli for most of the mammalian tissue varies in the range $50-10000 P a$ spanning from the softest tissue such as brain, retina, mammary glands to the stiffest tissue such as muscles, cartilage [78, 79, 80]. This yields $K_{w} \sim 10-10000$. We have explored here the range $10 \leqslant K_{w} \leqslant 10000$ and $0.05 \leqslant C \leqslant 1$ respectively. We set dimensionless active force, $S=10$, such that swimmer has sufficient time to assume a saturated shape in response to the imposed active force and excess perimeter, $\Gamma=0.085$. 


\section{Results and discussion}

The objective of this study is to investigate the effects of flexible walls on swimming hydrodynamics. The swimmer swims with its axis of symmetry along centerline. We first consider the instantaneous behavior and then the time averaged dynamics over a swimming cycle. The results are extracted after several initial swimming strokes once all transient dynamics have completely decayed.

\subsection{Instantaneous behavior within a swimming cycle}

\subsubsection{Swimmer shape evolution}

The non reciprocal cyclic shape changes exhibited by the axially moving amoeboid swimmer in flexible channel of confinement $C=0.8$ and wall stiffness $K_{w}=10$, during swimming cycle are shown in figure 2 . The amoeboid swimmer displays deformation based propulsion mechanism that involves formation of protrusion at the front of the body which propagates forward and then rearward resulting in a net forward motion as described in [35, 37]. The swimming cycle is broadly composed of four phases, namely, oblate, pusher, prolate and puller (see swimmer shapes in figure 2). This categorization is based on changes in swimmer shapes, flow pattern and corresponding instantaneous stresslet as discussed in earlier studies [37]. In brief, the swimmer with protrusion at the front end and dimple at the rear end that pushes fluid out from its anterior and posterior ends and pulls fluid towards its sides with positive instantaneous stresslet corresponds to the pusher while mirror image of shape of the pusher that pushes fluid out from sides of the swimmer and pulls in fluid to its ends with negative instantaneous stresslet corresponds to the puller (see flow field for pusher and puller in figure 5 (b) and (d) respectively).

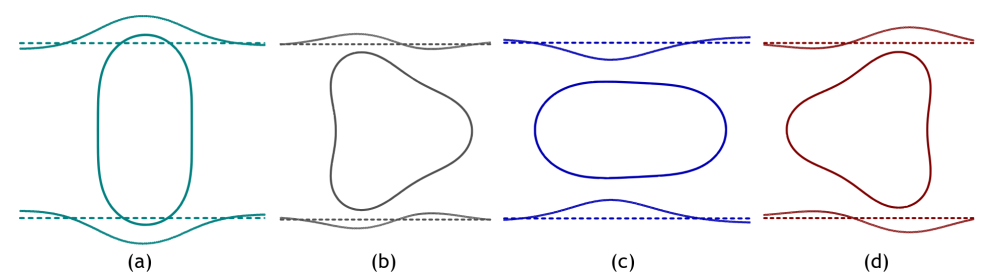

Figure 2: (color online) Temporal snapshots of shape changes, namely, (a) oblate, (b) pusher, (c) prolate and (d) puller, displayed by the axially moving amoeboid swimmer in flexible channel of confinement $C=0.8$ and wall stiffness $K_{w}=10$ over one swimming stroke.

\subsubsection{Analysis of the swimmer and wall deformation}

In order to understand swimmer behavior in flexible channel, we first analyse morphological features of the swimmer and flexible walls. Figure 3 (a) shows the temporal evolution of the lateral elongation, $D_{y}$, of the swimmer during two 
swimming strokes for different wall stiffnesses, $K_{w}$ (along with the corresponding swimmer shapes). The lateral deformation, $D_{y}$, measures the extension of the swimmer in the direction perpendicular to the flow (see schematics in figure $1)$. The maximum lateral extension over a swimming cycle is represented by $D_{y}^{\max }$. Large wall stiffness $\left(K_{w} \rightarrow \infty\right)$ restricts lateral extension of the swimmer whereas extremely flexible walls $\left(K_{w} \rightarrow 0\right)$ allow swimmer to freely exhibit shape changes. The highly flexible walls comply with the shape changes exhibited by the swimmer and display large wall deformation (see figure 2).

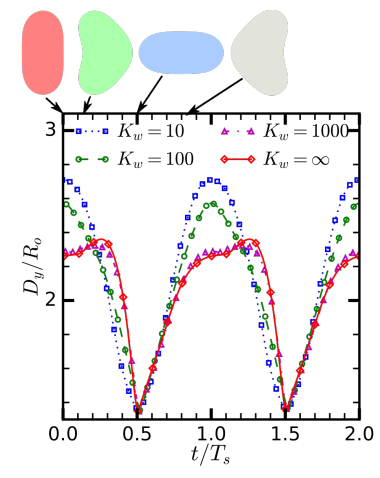

(a)

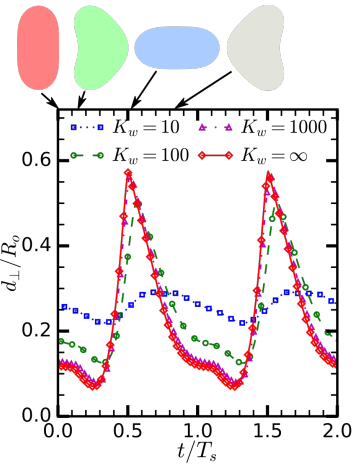

(b)

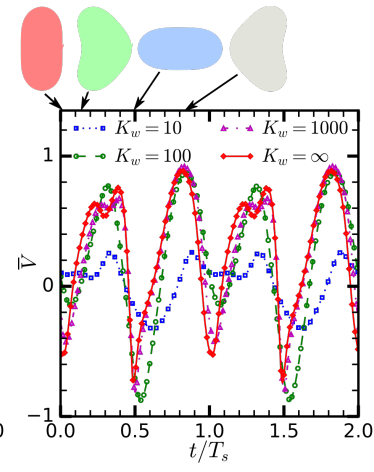

(c)

Figure 3: (color online) Temporal variation in (a) lateral deformation, $D_{y} / R_{o}$, of swimmer, (b) film thickness between swimmer and channel walls, $d_{\perp} / R_{o}$, and (c) instantaneous velocity, $\bar{V}=V T_{s} / R_{o}$, of an axially moving amoeboid swimmer in flexible channels of confinement $C=0.8$ and wall stiffnesses $K_{w}=$ $10,100,1000$ and $\infty$ over two swimming strokes. The variation of the swimmer shapes with time are shown at the top of the figures.

\subsubsection{Liquid film between the swimmer and channel walls}

The film thickness between swimmer and flexible channel wall is denoted by $d_{\perp}$. Figure 3 (b) shows temporal variation of the film thickness, $d_{\perp}$, for different wall deformabilities during two swimming periods (along with the corresponding swimmer shapes). The minimum and maximum film thickness over one swimming cycle are denoted as $d_{\perp}^{\min }$ and $d_{\perp}^{\max }$ respectively so that the amplitude of variation in film thickness over one swimming cycle is $d_{\perp}^{a m p}=d_{\perp}^{\max }-d_{\perp}^{\min }$. The film thickness displays sharp variation in the stiffer channel $\left(K_{w} \rightarrow \infty\right)$ compared to the flexible channel $\left(K_{w} \rightarrow 0\right)$. The flexible walls adapt according to the swimmer shapes and allow the swimmer to freely express its lateral extension, $D_{y}$, resulting in a minor variation in the film thickness, $d_{\perp}$, over swimming stroke. In contrast, stiffer channel obstructs lateral elongation of the swimmer and facilitates large variation in the film thickness between swimmer and channel walls over swimming stroke. The peak in $d_{\perp}$ curve represents prolate shape with large film thickness. The smaller film thickness is obtained for the pusher 
and oblate shape. A rigid channel $\left(K_{w}=\infty\right)$ displays sharp increase in the film thickness, $d_{\perp}$, during transition from pusher to prolate shape and facilitates dragging of large fluid mass rearwards while flexible channel $\left(K_{w}=10\right)$ due to its adaptability to the swimmer shape shows minor increase in the film thickness displacing thus a smaller fluid volume rearwards. The minimum film thickness, $d_{\perp}^{\text {min }}$, in the flexible channel is large compared to that in the rigid channel allowing forward leakage of the fluid that was dragged rearward (see variation in $d_{\perp}$ for the flexible channel $\left(K_{w}=10\right)$ in figure $\left.3(\mathrm{~b})\right)$. The influence of the film thickness, $d_{\perp}$, and its variation, $d_{\perp}^{a m p}$, on the swimmer motion is further discussed in sections 3.2.3 and 3.3.

\subsubsection{Swimmer motion and flow field}

The temporal variation of the instantaneous velocity, $\bar{V}(t)$, of the swimmer for different wall stiffnesses over two swimming cycles (along with the corresponding swimmer shapes) is shown in figure 3 (c). The dimensional instantaneous swimmer velocity, $V$, is scaled to obtain dimensionless instantaneous swimmer velocity, $\bar{V}=V T_{s} / R_{o}$. A general outcome is the decline of the swimmer velocity due to wall compliance. This may seem a priori surprising since rigid channel restricts lateral extension of the swimmer, precluding thus the swimmer to fully deploy its amoeboid latitude. The physical interpretation of this observation is given in section 3.3. Figure 4 shows comparison of the instantaneous swimmer velocity in flexible $\left(K_{w}=10\right)$ and rigid $\left(K_{w}=\infty\right)$ channels. In highly flexible channel $\left(K_{w} \sim 10\right)$, weaker forward motion is exhibited during pusher and oblate phase while rearward motion is displayed in the puller phase. In stiffer channel $\left(K_{w} \rightarrow \infty\right)$, strong forward motion is exhibited during puller and pusher phases while comparatively weaker backward motion is displayed by the oblate and the prolate swimmer resulting in a strong net forward motion of the swimmer.

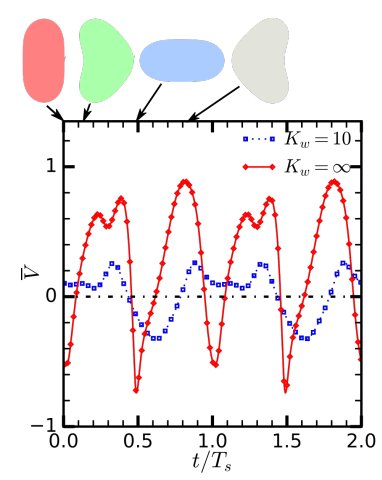

Figure 4: (color online) Temporal variation of the instantaneous swimmer velocity, $\bar{V}=V T_{s} / R_{o}$, in flexible $\left(K_{w}=10\right)$ and rigid $\left(K_{w}=\infty\right)$ channels of confinement $C=0.8$ over two swimming strokes. 
The comparison of instantaneous flow field is shown in figure 5 by superimposing velocity vector field over velocity contours. As discussed in section 3.1.1 and figure 2, the swimming stroke comprised of four phases, namely, oblate, pusher, prolate and puller.

- In the oblate phase, the swimmer in the flexible channel (see figure 5 (a)) shows weaker forward velocity compared to the rigid channel (compare figures 5 (a) and (e)). In stiff channel, fluid along sides of the oblate swimmer close to the walls moves rearward (see figure 5 (e)) while the fluid inside the swimmer moves forward. However, the net swimmer motion is rearward. This contrasts with flexible channel case (see figure 5 (a)) where swimmer moves forward (see instantaneous swimmer velocity for the oblate swimmer in flexible and rigid channels in figure 4). In other words, flexible channel facilitates motion of the oblate swimmer.

- The pusher in the rigid channel (see figure 5 (f)) shows forward velocity field at its front tip and very weak rearward velocity field at its rear dimpled end resulting in a strong net forward motion. The pusher in the flexible channel (see figure 5 (b)) displays weaker forward motion (due to presence of strong rearward velocity field at its rear end; compare instantaneous velocity of pusher in flexible and rigid channels in figure 4). In

this regime, when lateral extension of the swimmer is large, the channel flexibility does not allow the swimmer to efficiently grasp on the wall to move forward. Thus flexibility in this phase unfavors swimmer motion.

- The prolate swimmer in the rigid channel exhibits strong backward motion (see figure $5(\mathrm{~g})$ ) compared to the soft channel (see figure 5 (c); compare instantaneous velocity of the prolate swimmer in flexible and rigid channels in figure 4). The rigid channel unfavors swimmer motion.

- Finally, in the puller phase, the swimmer in the rigid channel (see figure 5 (h)) exhibits significant forward motion contrary to the flexible channel (see figure 5 (d)) where it shows net rearward motion (see instantaneous velocity of the puller in flexible and rigid channels in figure 4). In this phase, wall flexibility again reduces grasping of the swimmer on the wall.

\subsubsection{Flow history}

The velocity vector field and contour plot provide instantaneous description of the flow field. It provides little information about history of the flow. Thus, in order to distinguish between the flow behaviour in rigid and flexible channels, we introduced massless tracer particles in the flow field and examined their flow pattern over several swimming strokes. Figure 6 shows comparison of evolution of tracer particles in rigid and flexible channels. The evolution of tracer particles for an unconfined swimmer $(C=0)$ is shown in figure 7. In figures 6 and 7 , subfigures (a) and (d) show initial and (b) and (e) display final positions of tracer particles after single swimming cycle, while subfigures (c) and (f) show 
positions of tracer particles after five swimming cycles. The particles in each column are colored differently to facilitate tracking of position of each particle. As shown in subfigures 6 (a) and (d), the tracer particles along sides of the swimmer whose motion is to be tracked are enclosed by the red boundary. These tracer particles in flexible and rigid channels are displaced rearwards (see subfigures 6 (b) and (e)). The tracer particles in flexible channel display significantly smaller displacement in rearward direction while tracer particles in rigid channel show large backward displacement (compare displacement and position of the tracer particles enclosed by the red boundary in subfigures 6 (b) and (e) for rigid and flexible channels respectively). This shows that there is strong net reverse flow through the film between swimmer and channel walls in rigid channel compared to the flexible channel. In the case of an unconfined swimmer, a small layer of tracer particles along sides of the swimmer experiences forward drag due to swimmer forward motion while shape changes exhibited by the swimmer displaces fluid mass which is slightly away from the swimmer surface rearwards (compare displacement and position of the tracer particles in subfigures 7 (a), (b) and (c)). The fluid far away from the unconfined swimmer remains unaffected.

The active force imposed on the swimmer is responsible for shape changes in the swimmer, its deformation, $D_{y}$, and variation in the film thickness, $d_{\perp}$, between swimmer and channel walls over swimming stroke which in turn play crucial role in trapping and dragging of fluid mass rearwards and obstructing its forward leakage (see variation in $D_{y}$ and $d_{\perp}$ over swimming stroke in figures 3 (a) and (b) respectively). The significant pressure drag developed across the swimmer and sharp variation in the film thickness, $d_{\perp}$, over swimming period for the swimmer in rigid channel utilizes viscous effects at the walls to facilitate entrapping and rearward dragging of large fluid volume through the film between swimmer and channel walls while smaller minimum film thickness, $d_{\perp}^{\text {min }}$, prevents its forward leakage (see $d_{\perp}$ in figure $3(\mathrm{~b})$ ). In contrast to rigid channel, flexible walls comply with changes in swimmer shapes yielding weaker pressure drag across the swimmer and small variation in the film thickness (roughly constant film thickness) over swimming cycle drives smaller fluid volume rearwards as it allows forward leakage due to larger minimum film thickness (see variation in film thickness, $d_{\perp}$, in flexible $\left(K_{w}=10\right)$ and rigid $\left(K_{w}=\infty\right)$ channel in figure $3(\mathrm{~b})$ ). (See rearward displacement of tracer particles in rigid and flexible channels in figure 6 ). In brief, the presence of rigid walls facilitates swimmer to induce stronger net reverse flow through the film between swimmer and channel walls compared to deformable walls.

\subsection{Time averaged dynamics over a swimming cycle}

In this section, we study the effects of wall deformability on the time averaged behaviour of the swimmer in a flexible channel over one swimming cycle. 


\subsubsection{Time averaged swimmer velocity}

The swimmer motion is characterized by its average velocity over a swimming period, $T_{s}$. The average swimmer velocity is defined as displacement of centroid of the swimmer over one swimming cycle. The dimensionless instantaneous velocity and displacement are $\bar{V}=V T_{s} / R_{o}$ and $\bar{X}_{c}=X_{c} / R_{o}$ respectively where $X_{c}$ is center of mass of the swimmer. The dimensionless time averaged velocity is $\langle\bar{V}\rangle=\left\langle\bar{X}_{c}\left(t+T_{s}\right)-\bar{X}_{c}(t)\right\rangle$. Figure 8 shows variation of time averaged swimmer velocity, $\langle\bar{V}\rangle$, with confinement, $C$, in deformable channels for different wall stiffnesses, $K_{w}$. The flexible channel of wall stiffness, $K_{w} \rightarrow \infty$, quantitatively reproduces results corresponding to the rigid channel study of $\mathrm{Wu}$ et al. [37]. The swimmer velocity in stiffer channel $\left(K_{w} \geqslant 100\right)$ initially increases for weak confinement reaching maximum magnitude for an optimum confinement and then decreases for higher confinements. Wu et al. [37] also showed that amoeboid swimmer bounded between rigid walls exhibits nonmonotonous variation in velocity with confinement. Earlier studies in the literature for non amoeboid swimmer $[81,82,83,84,85]$ showed enhancement or a decline of swimmer speed due to confinement.

The swimmer velocity decreases with wall softness (compare $\langle\bar{V}\rangle$ for different wall stiffnesses in figure 8$)$. Extremely soft channel $\left(K_{w}=10\right)$ shows monotonic decrease in the swimmer velocity with the confinement. Eventually at high confinement $(C \sim 1)$ and very small wall stiffness $\left(K_{w} \sim 10\right)$, the swimmer is trapped and is unable to move forward. We will discuss potential causes for such swimming dynamics in rigid and flexible channels in section 3.3.

\subsubsection{Deformation of the swimmer and the walls}

Figures 9 (a) and (b) show maximum deformation of the wall, $\left|\delta_{w}\right|^{\text {max }}$, and maximum lateral deformation of the swimmer, $D_{y}^{\max }$, with confinement, $C$, over one swimming cycle for different wall stiffnesses. The wall deformation, $\left|\delta_{w}\right|$, is computed as the vertical deviation of the wall position from its initial reference configuration, and is defined as $\left|\delta_{w}\right|=\left|\mathbf{x}_{\text {def }}-\mathbf{x}_{r e f}\right|$. Here $\mathbf{x}_{r e f}$ and $\mathbf{x}_{\text {def }}$ represents undeformed (reference) and deformed configuration (see figure 1 ). The maximum wall deformation over one swimming period is expressed as $\left|\delta_{w}\right|^{\text {max }}$. As discussed earlier, strong confinement of rigid channel $\left(K_{w}=\infty\right)$ restricts swimmer's lateral elongation but allows its axial elongation whereas flexible walls $\left(K_{w}=10\right)$ deform as per swimmer shape and allow its complete lateral elongation. Thus the swimmer moving through highly flexible channel can exhibits shape changes similar to an unconfined swimmer.

\subsubsection{Film thickness}

The variation in minimum film thickness, $d_{\perp}^{m i n}$, and amplitude of variation in film thickness, $d_{\perp}^{a m p}=d_{\perp}^{\max }-d_{\perp}^{\text {min }}$, between swimmer and channel walls, are shown in figures 10 (a) and (b) respectively. The increase in confinement results in a decrease in minimum film thickness and reduction in amplitude of variation in film thickness. The minimum film thickness, $d_{\perp}^{\text {min }}$, for the flexible 
channel is higher than that of the rigid channel. In contrasts, the amplitude of variation in film thickness, $d_{\perp}^{a m p}$, in flexible channel is smaller than that in the rigid channel for the same confinement due to adaptability of flexible walls to the swimmer shapes. The role played by the minimum film thickness and amplitude of variation in film thickness over swimming stroke in controlling flow dynamics and propulsion speed of the swimmer in rigid and flexible channels is discussed in section 3.3.

\subsubsection{Stresslet, power consumption and swimming efficiency}

The nature of the swimmer either pusher or puller is defined based on the stresslet. The instantaneous dimensionless stresslet is defined as $\bar{\Sigma}=\left(\sigma_{x x}-\sigma_{y y}\right) /\left(\eta R_{o}^{2} / T_{s}\right)$, $\sigma_{i j}=\oint_{\Omega_{3}} f_{i} r_{j} d s$. The negative stresslet corresponds to the puller whereas positive stresslet represents pusher. During single swimming stroke, amoeboid swimmer switches between puller and pusher nature. Thus average nature of the swimmer (pusher or puller) over swimming cycle is obtained by time averaging of the stresslet over swimming period. The time averaged stresslet is expressed as $\langle\bar{\Sigma}\rangle=\left(\int_{0}^{T_{s}} \bar{\Sigma} d t\right) / T_{s}$. The variation of time averaged stresslet, $\langle\bar{\Sigma}\rangle$, with confinement over one swimming cycle for different wall stiffnesses is shown in figure 11 (a). The swimmer in high confinement rigid channel displays strong time averaged puller behaviour while it shows comparatively weaker time averaged puller nature in the flexible channel.

The power consumed by the swimmer over one swimming cycle is evaluated as follows [37].

$$
\left\langle P_{s}\right\rangle=\frac{1}{T_{s}} \int_{0}^{T_{s}} \oint_{\Omega_{3}}(\mathbf{f}(s) \cdot \mathbf{u}(s)) d s d t=\left\langle\oint_{\Omega_{3}}(\mathbf{f}(s) \cdot \mathbf{u}(s)) d s\right\rangle,
$$

where $P_{s}$ is time averaged power consumption, $T_{s}$ is time period of a swimming cycle and $\langle\cdot\rangle$ represents time averaged quantities. Figure 11 (b) shows variation of dimensionless average power, $\left\langle\bar{P}_{s}\right\rangle=\left\langle P_{s}\right\rangle T_{s}^{2} /\left(\eta R_{o}^{2}\right)$, with confinement for different wall stiffnesses. The power consumed by the swimmer is reduced due to presence of softer walls $\left(K_{w} \rightarrow 10\right)$. The power consumption in stiffer channel $\left(K_{w}=\infty\right)$ is maximum at the confinement slightly higher than the confinement where maximum swimmer velocity is attained whereas for very soft channel $\left(K_{w}=10\right)$, power consumed by the swimmer remains roughly constant. The power consumption alone does not provide significant information about the swimmer performance. Thus it is crucial to analyse swimmer efficiency. The swimmer efficiency, $\Pi$, can be defined as the ratio of least power consumed to displace swimmer with its time averaged velocity to the swimmer's actual time averaged power consumption as discussed in $[37,86,87,88] . \Pi=\eta\langle\bar{V}\rangle^{2} /\left\langle\overline{P_{s}}\right\rangle$. Figure $11(\mathrm{c})$ shows swimmer efficiency, $\Pi$, as a function of confinement, for different wall stiffnesses. The amoeboid swimmer in stiffer channel shows maximum efficiency for the confinement slightly lower than the confinement corresponding to maximum swimmer velocity. The swimmer efficiency drops in presence of flexible walls. The swimmer in softer channel $\left(K_{w}=10\right)$ displays 
sharp decrease in efficiency with increase in the confinement strength. This shows that amoeboid swimmer is inefficient in presence of deformable walls.

\subsection{Swimming mechanism in confined channel}

The study of instantaneous flow field and history of the flow by tracing motion and trajectory of fluid particles showed that amoeboid swimmer utilizes two complementary mechanisms that govern its propulsion speed in confined channel. They comprise of swimmer propulsion due to imposed active force complemented by purely hydrodynamic means which involves utilizing shape changes exhibited by the swimmer and viscous drag along the walls to drive fluid mass rearwards from front of the swimmer. The forward motion of swimmer in a stagnant fluid requires swimmer to develop a passage for its locomotion by displacing fluid mass present in its anterior region and driving this displaced fluid rearward which then occupy the posterior space vacated due to swimmer mobility.

\subsubsection{Swimming in weakly confined channel}

In an unconfined and weakly confined channel $(C \leqslant 0.25)$, the swimmer is far away from the walls and the minimum film thickness, $d_{\perp}^{\text {min }}$, between the swimmer and channel walls is large (see minimum film thickness, $d_{\perp}^{\text {min }}$, for the confinement of $C \leqslant 0.25$ in figure 10 (a)). Thus the swimmer motion is not affected by the channel walls and the swimmer moves with a constant time averaged velocity (see time averaged velocity, $\langle\bar{V}\rangle$, of the swimmer for confinements, $C \leqslant 0.25$, in figure 8). The swimmer forward motion in a weakly confined channel pushes the fluid mass present in front of the swimmer resulting in a free lateral motion of the fluid particles as shown in figure 7 for an unconfined swimmer. The shape changes exhibited by the swimmer propels this fluid mass rearwards along its sides (see rearward displacement of tracer particles for an unconfined swimmer after five swimming periods in figure 7 (c)).

\subsubsection{Swimming in confined rigid channel}

As the channel confinement increases, the minimum film thickness, $d_{\perp}^{\text {min }}$, between the swimmer and plane rigid wall decreases and the viscous drag along rigid wall increases, influencing thus the flow field in the vicinity of the swimmer. The shape changes displayed by the swimmer induces variation in the film thickness, $d_{\perp}$, over swimming cycle and utilizes viscous drag along the channel walls to facilitate trapping of the fluid mass from the front of the swimmer within the film between the swimmer and channel walls, dragging it rearward and obstructing its forward leakage.

In moderately confined rigid channels $(0.4 \leqslant C \leqslant 0.7)$, the minimum film thickness, $d_{\perp}^{\min }$, between the swimmer and the channel walls is modest and

thus allows large lateral deformation of the swimmer, $D_{y}^{\max }$, and corresponding large amplitude of variation in film thickness, $d_{\perp}^{a m p}$, over swimming cycle (see 
variation of $D_{y}^{\max }, d_{\perp}^{\min }$ and $d_{\perp}^{a m p}$ for moderately confined rigid channels in figures 9 (b), 10 (a) and (b) respectively). The cyclic non reciprocal swimmer shape changes utilize the viscous drag along rigid walls and variation in the film thickness to accelerate rearward transport of a larger fluid volume. Thus the swimmer forward motion imposed by the active force is complemented by an increase in the net reverse flow rate of the fluid through the film between the swimmer and the channel walls with an increase in confinement in moderately confined rigid channels resulting in an enhancement of the swimmer velocity (see figure 8).

The maximum swimmer velocity is attained in rigid channels for a confinement of $C \sim 0.7$ (see figure 8) with an optimum minimum film thickness that slightly obstructs the lateral elongation of the swimmer. This helps swimmer to efficiently utilize viscous drag developed at the rigid walls to drive maximum rearward flow through the film while inhibiting its forward leakage.

In strongly confined rigid channels $(C \geqslant 0.8)$, substantial pressure drag develops across the swimmer. The minimum film thickness between the swimmer and channel walls reduces and the rigid walls restrict lateral deformation of the swimmer resulting in small amplitude of variation in the film thickness over swimming cycle (see reduction in variation in $D_{y}^{\max }$ and $d_{\perp}^{a m p}$ for strongly confined rigid channel $(C \geqslant 0.8)$ in figures 9 (b) and 10 (b) respectively). The swimmer can not propel enough fluid volume rearwards through the thin film between the swimmer and channel walls against strong viscous drag developed along rigid walls and swimmer surface. This results in a significant reduction in swimmer velocity in highly confined rigid channel $(C \geqslant 0.8)$ (see figure 8).

\subsubsection{Swimming in confined flexible channel}

The highly flexible channel $\left(K_{w}=10\right)$ allows the swimmer to freely exhibit shape changes imposed by the active force and display large lateral elongation for all confinements (maximum lateral elongation, $D_{y}^{\max }$, of the swimmer in flexible channel $\left(K_{w}=10\right)$ for all confinement is constant as shown in figure 9 (b)). The minimum film thickness between the swimmer and flexible walls, which slowly reduces with an increase in confinement in soft channel, is still larger compared to the rigid channel for the same confinement strength (compare $d_{\perp}^{\text {min }}$ for rigid $\left(K_{w}=\infty\right)$ and flexible channel $\left(K_{w}=10\right)$ in figure $\left.10(\mathrm{a})\right)$. As the flexible walls adapt their configuration according to the swimmer shapes, the amplitude of variation in film thickness over a swimming period sharply reduces with an increase in confinement (see reduction in $d_{\perp}^{a m p}$ in flexible channel $\left(K_{w}=10\right)$ in figure $\left.10(\mathrm{~b})\right)$. The small amplitude of variation in film thickness over swimming period can trap and drag smaller fluid volume rearwards and allows considerable forward leakage due to larger minimum film thickness. The rearward displacement of the fluid mass is reduced resulting in a decrease in net reverse flow rate through the film with an increase in confinement. As the fluid flow through the film becomes sluggish, the frictional drag within the film retards the swimmer forward motion imposed by the active force. This causes reduction in the swimmer velocity with an increase in confinement in flexible 
channel (see results for $\langle\bar{V}\rangle$ for $\left(K_{w}=10\right)$ in figure 8).

In strongly confined flexible channel, smaller film thickness with its negligible variation over swimming stroke (see $d_{\perp}^{\text {min }}$ and $d_{\perp}^{a m p} \rightarrow 0$ in flexible channels ( $\left.K_{w}=10\right)$ for confinements of $C>0.8$ in figures 10 (a) and (b) respectively) develops larger viscous drag along flexible walls and swimmer surface compared to the weaker confinement. This hinders net rearward fluid flow through the film resulting in a substantial deceleration of the swimmer forward motion in strong confinement. The active force merely serves to displace the channel walls than to transfer momentum to the swimmer, so that the swimmer is trapped at its initial position (see swimmer velocity, $\langle\bar{V}\rangle$, in flexible channel $\left(K_{w}=10\right)$ of confinement $C=1$ in figure 8).

\section{Conclusion}

The current two dimensional numerical study investigated the effects of wall flexibility and channel confinement on the motion of an amoeboid microswimmer through the flexible channel. The time averaged velocity of the swimmer in flexible channels is lower than that in a rigid channel. The swimmer velocity in flexible channels decreases substantially with an increase in confinement whereas the swimmer velocity in stiffer channel displays nonmonotonic variation. The strongly confined soft channel severely obstructs forward motion of the swimmer and imprisons it.

Such reduction in swimming speed with an increase in wall flexibility is also observed in numerical studies by Fauci and McDonald [59] and Shaik et al. [60] who investigated spermatozoid and squirmer model respectively while LedesmaAguilar and Yeomans [58] reported increase in motility of dipolar swimmer. In view of these results and the present work, it seems that the nature of the swimmer may play a decisive role. The same conclusion is reached while analyzing the role of confinement by rigid walls. The confinement is found to enhance or decelerate the swimmer motion depending on the nature of the swimmer $[81,82,83,84,36]$. A recent analytical calculation has confirmed this conclusion [89]. This highlights the subtle character of wall effects on motility of the swimmer.

In this study, we explored the role of dimensionless wall stiffness on the swimming dynamics. It will be interesting to investigate the impact of other parameters such as dimensionless active force and confinement on the swimming dynamics in future studies in order to explore full potential of the present model. Our earlier three dimensional studies on an amoeboid swimming [35, 90] did not reveal any new interesting features as compared to the two dimensional studies [36, 37]. Thus we performed two dimensional investigation in this article. Nevertheless, three dimensional study will be explored in the future. We consider viscosity of enclosing and suspending fluid to be equal for simplification. Our previous study [90] investigated effects of viscosity contrast on the swimming dynamics but did not divulge any new findings. The enclosing and suspending fluid and cell cortex act as a dashpots in parallel and are responsible 
for dissipation within the system. In other words, swimming features depend on the effective viscosity of the system which is linear combination of these three viscosities (see [91]).

We used here a simplified plate spring type model for modeling the flexible walls (described in section 2.3). But flexible walls representing real media (such as tissues) can be accurately represented as continuum elastic solid with dissipative effects. These elastic solids can be modeled as either incompressible linearized elastic solids with dissipative viscous effects for low strains or incompressible neo Hookean viscoelastic solids for large strains. More realistic models should be considered in future studies to check the impact of the complexity of the model on the main results. Finally, the walls have been considered to be impermeable. In the case of swimmer motion through tissues, this hypothesis ceases to be valid and the effect of permeation to liquid may add an extra level of complexity. It is hoped to investigate this matter further in future studies.

\section{Appendix: Justification for selection of nor- mal active force}

In this appendix, we justify assumption of considering active force acting along the normal direction. Let's consider active force comprising of both normal and tangential components and is expressed as,

$$
\mathbf{f}_{a}=f_{a n} \mathbf{n}+f_{a t} \mathbf{t} .
$$

The objective here is to show that we are at liberty to choose new active force distribution, $\mathbf{f}_{a}^{\prime}=f_{a n}^{\prime} \mathbf{n}$, which acts purely along normal direction without affecting total force, $\mathbf{f}$, that is the new total force acting along the swimmer membrane is equal to the old one, $\mathbf{f}^{\prime}=\mathbf{f}$.

From equations (6) and (3), the total force acting along the swimmer membrane is written as,

$$
\mathbf{f}=\mathbf{f}_{a}+\mathbf{f}_{0}+\widetilde{f}_{0} \mathbf{t}-c \zeta \mathbf{n}+\frac{\partial \zeta}{\partial s} \mathbf{t}
$$

For simplicity, the bending force is ignored from total active force expression as it is purely normal component and does not play any role in the current discussion. Similarly, new total force, $\mathbf{f}^{\prime}$, is expressed as,

$$
\mathbf{f}^{\prime}=\mathbf{f}_{a}^{\prime}+\mathbf{f}_{0}^{\prime}+{\widetilde{f_{0}^{\prime}}}_{0} \mathbf{t}-c \zeta^{\prime} \mathbf{n}+\frac{\partial \zeta^{\prime}}{\partial s} \mathbf{t}
$$

We impose that the old and new total forces are equal, $\mathbf{f}=\mathbf{f}^{\prime}$, and compare their normal and tangential components.

$$
\begin{array}{r}
f_{a n}+\mathbf{f}_{0} \cdot \mathbf{n}-c \zeta=f_{a n}^{\prime}+\mathbf{f}_{0}^{\prime} \cdot \mathbf{n}-c \zeta^{\prime} \\
f_{a t}+\mathbf{f}_{0} \cdot \mathbf{t}+\widetilde{f}_{0}+\frac{\partial \zeta}{\partial s}=\mathbf{f}_{0}^{\prime} \cdot \mathbf{t}+\widetilde{f}_{0}^{\prime}+\frac{\partial \zeta^{\prime}}{\partial s}
\end{array}
$$


We integrate last equation over swimmer perimeter and use definition of tangent to the curve, $\mathbf{t}=\frac{\partial \mathbf{r}}{\partial s}$, where $\mathbf{r}$ is the position of a point on the membrane, to obtain,

$$
\widetilde{f}_{0}^{\prime}=\widetilde{f}_{0}+\frac{\oint f_{a t} d s}{\oint d s} .
$$

We use results from equation (14) in equation (13) to derive following expressions.

$$
\begin{aligned}
\zeta^{\prime} & =\int_{0}^{s} f_{a t}\left(s^{\prime}\right) d s^{\prime}+\left(\mathbf{f}_{0}-\mathbf{f}_{0}^{\prime}\right) \cdot \mathbf{r}-\frac{\oint f_{a t} d s^{\prime}}{\oint d s^{\prime}} s+\zeta, \\
f_{a n}^{\prime} & =f_{a n}+\left(\mathbf{f}_{0}-\mathbf{f}_{0}^{\prime}\right) \cdot \mathbf{n}-c\left(\zeta-\zeta^{\prime}\right) .
\end{aligned}
$$

This shows that redefinition of the Lagrange multiplier and normal active force allows us to rewrite active force expression in terms of a normal component only.

\section{Conflicts of interest}

There are no conflicts to declare.

\section{Acknowledgements}

We thank CNES (Centre National d'Etudes Spatiales) and the French-German University Programme "Living Fluids" (Grant No. CFDA-Q1-14) for financial support.

\section{References}

[1] Mitchell L Sogin, Hilary G Morrison, Julie A Huber, David Mark Welch, Susan M Huse, Phillip R Neal, Jesus M Arrieta, and Gerhard J Herndl. Microbial diversity in the deep sea and the underexplored rare biosphere. Proc. Natl. Acad. Sci., 103(32):12115-12120, 2006.

[2] William B Whitman, David C Coleman, and William J Wiebe. Prokaryotes: the unseen majority. Proc. Natl. Acad. Sci., 95(12):6578-6583, 1998.

[3] Thomas P Curtis, William T Sloan, and Jack W Scannell. Estimating prokaryotic diversity and its limits. Proc. Natl. Acad. Sci., 99(16):1049410499, 2002.

[4] Inna Sekirov, Shannon L Russell, L Caetano M Antunes, and B Brett Finlay. Gut microbiota in health and disease. Physiol. Rev., 90(3):859904, 2010. 
[5] Tara J Moriarty, M Ursula Norman, Pina Colarusso, Troy Bankhead, Paul Kubes, and George Chaconas. Real-time high resolution 3d imaging of the lyme disease spirochete adhering to and escaping from the vasculature of a living host. PLoS Pathogens, 4(6):e1000090, 2008.

[6] Howard A Stone, Abraham D Stroock, and Armand Ajdari. Engineering flows in small devices: microfluidics toward a lab-on-a-chip. Annu. Rev. Fluid Mech., 36:381-411, 2004.

[7] Jamil El-Ali, Peter K Sorger, and Klavs F Jensen. Cells on chips. Nature, 442(7101):403, 2006.

[8] Sussan Nourshargh, Peter L Hordijk, and Michael Sixt. Breaching multiple barriers: leukocyte motility through venular walls and the interstitium. Nat. Rev. Mol. Cell Biol., 11(5):366, 2010.

[9] Bradley J Nelson, Ioannis K Kaliakatsos, and Jake J Abbott. Microrobots for minimally invasive medicine. Annu. Rev. Biomed. Eng., 12:55-85, 2010.

[10] Li Zhang, Kathrin E Peyer, and Bradley J Nelson. Artificial bacterial flagella for micromanipulation. Lab Chip, 10(17):2203-2215, 2010.

[11] Kathrin E Peyer, Li Zhang, and Bradley J Nelson. Bio-inspired magnetic swimming microrobots for biomedical applications. Nanoscale, 5(4):12591272, 2013.

[12] Eric Lauga, Willow R DiLuzio, George M Whitesides, and Howard A Stone. Swimming in circles: motion of bacteria near solid boundaries. Biophys. J., 90(2):400-412, 2006.

[13] Howard C Berg and Linda Turner. Chemotaxis of bacteria in glass capillary arrays. escherichia coli, motility, microchannel plate, and light scattering. Biophys. J., 58(4):919-930, 1990.

[14] Daniela Pimponi, Mauro Chinappi, Paolo Gualtieri, and Carlo Massimo Casciola. Hydrodynamics of flagellated microswimmers near free-slip interfaces. J. Fluid Mech., 789:514-533, 2016.

[15] R Di Leonardo, D Dell'Arciprete, L Angelani, and V Iebba. Swimming with an image. Phys. Rev. Lett., 106(3):038101, 2011.

[16] Laurence Lemelle, Jean-François Palierne, Elodie Chatre, and Christophe Place. Counterclockwise circular motion of bacteria swimming at the airliquid interface. J. Bacteriol., 192(23):6307-6308, 2010.

[17] Luis Cisneros, Christopher Dombrowski, Raymond E Goldstein, and John O Kessler. Reversal of bacterial locomotion at an obstacle. Phys. Rev. E, 73(3):030901, 2006. 
[18] Michael Morse, Athena Huang, Guanglai Li, Martin R Maxey, and Jay X Tang. Molecular adsorption steers bacterial swimming at the air/water interface. Biophys. J., 105(1):21-28, 2013.

[19] Howard A Barnes. A review of the slip (wall depletion) of polymer solutions, emulsions and particle suspensions in viscometers: its cause, character, and cure. J. Non-Newtonian Fluid Mech., 56(3):221-251, 1995.

[20] Diego Lopez and Eric Lauga. Dynamics of swimming bacteria at complex interfaces. Phys. Fluids, 26(7):400-412, 2014.

[21] Darren Crowdy, Sungyon Lee, Ophir Samson, Eric Lauga, and AE Hosoi. A two-dimensional model of low-reynolds number swimming beneath a free surface. J. Fluid Mech., 681:24-47, 2011.

[22] Allison P Berke, Linda Turner, Howard C Berg, and Eric Lauga. Hydrodynamic attraction of swimming microorganisms by surfaces. Phys. Rev. Lett., 101(3):038102, 2008.

[23] DJ Smith, EA Gaffney, JR Blake, and JC Kirkman-Brown. Human sperm accumulation near surfaces: a simulation study. J. Fluid Mech., 621:289$320,2009$.

[24] Davide Giacché, Takuji Ishikawa, and Takami Yamaguchi. Hydrodynamic entrapment of bacteria swimming near a solid surface. Phys. Rev. E, 82(5):056309, 2010.

[25] Guanglai Li, James Bensson, Liana Nisimova, Daniel Munger, Panrapee Mahautmr, Jay X Tang, Martin R Maxey, and Yves V Brun. Accumulation of swimming bacteria near a solid surface. Phys. Rev. E, 84(4):041932, 2011.

[26] Guillaume Charras and Ewa Paluch. Blebs lead the way: how to migrate without lamellipodia. Nat. Rev. Mol. Cell Biol., 9(9):730, 2008.

[27] Albert J Bae and Eberhard Bodenschatz. On the swimming of dictyostelium amoebae. Proc. Natl. Acad. Sci., 107(44):E165, 2010.

[28] Nicholas P Barry and Mark S Bretscher. Dictyostelium amoebae and neutrophils can swim. Proc. Natl. Acad. Sci., 107(25):11376-11380, 2010.

[29] Tim Lämmermann, Bernhard L Bader, Susan J Monkley, Tim Worbs, Roland Wedlich-Söldner, Karin Hirsch, Markus Keller, Reinhold Förster, David R Critchley, Reinhard Fässler, et al. Rapid leukocyte migration by integrin-independent flowing and squeezing. Nature, 453(7191):51, 2008.

[30] Laurene Aoun, Paulin Negre, Alexander Farutin, Nicolas Garcia-Seyda, Mohd Suhail Rivzi, Remi Galland, Alphee Michelot, Xuan Luo, Martine Biarnes-Pelicot, Claire Hivroz, Salima Rafai, Jean-Baptiste Sibareta, Marie-Pierre Valignat, Chaouqi Misbah, and Olivier Theodoly. Mammalian amoeboid swimming is propelled by molecular and not protrusion-based paddling in lymphocytes. bioRxiv, page 509182, 2019. 
[31] Marino Arroyo, Luca Heltai, Daniel Millán, and Antonio DeSimone. Reverse engineering the euglenoid movement. Proc. Natl. Acad. Sci., 109(44):17874-17879, 2012.

[32] Jahn Throndsen. Flagellates of norwegian coastal waters. Nord. J. Bot., 16:161-216, 1969.

[33] Takao Ohta and Takahiro Ohkuma. Deformable self-propelled particles. Phys. Rev. Lett., 102(15):154101, 2009.

[34] Tetsuya Hiraiwa, Kyohei Shitara, and Takao Ohta. Dynamics of a deformable self-propelled particle in three dimensions. Soft Matter, 7(7):30833086, 2011.

[35] Alexander Farutin, Salima Rafaï, Dag Kristian Dysthe, Alain Duperray, Philippe Peyla, and Chaouqi Misbah. Amoeboid swimming: a generic selfpropulsion of cells in fluids by means of membrane deformations. Phys. Rev. Lett., 111(22):228102, 2013.

[36] Hao Wu, Marine Thiébaud, W-F Hu, Alexander Farutin, S Rafaï, M-C Lai, Philippe Peyla, and Chaouqi Misbah. Amoeboid motion in confined geometry. Phys. Rev. E, 92(5):050701, 2015.

[37] Hao Wu, Alexander Farutin, Wei-Fan Hu, Marine Thiébaud, Salima Rafaï, Philippe Peyla, Ming-Chih Lai, and Chaouqi Misbah. Amoeboid swimming in a channel. Soft Matter, 12(36):7470-7484, 2016.

[38] Rhoda J Hawkins, Matthieu Piel, G Faure-Andre, AM Lennon-Dumenil, JF Joanny, J Prost, and R Voituriez. Pushing off the walls: a mechanism of cell motility in confinement. Phys. Rev. Lett., 102(5):058103, 2009.

[39] Jérôme Lohéac, Jean-François Scheid, and Marius Tucsnak. Controllability and time optimal control for low reynolds numbers swimmers. Acta Appl. Math., 123(1):175-200, 2013.

[40] Jayant Pande and Ana-Sunčana Smith. Forces and shapes as determinants of micro-swimming: effect on synchronisation and the utilisation of drag. Soft Matter, 11(12):2364-2371, 2015.

[41] François Alouges, Antonio DeSimone, and Luca Heltai. Numerical strategies for stroke optimization of axisymmetric microswimmers. Math. Models Methods Appl. Sci., 21(02):361-387, 2011.

[42] Andrej Vilfan. Optimal shapes of surface slip driven self-propelled microswimmers. Phys. Rev. Lett., 109(12):128105, 2012.

[43] JE Avron, O Gat, and O Kenneth. Optimal swimming at low reynolds numbers. Phys. Rev. Lett., 93(18):186001, 2004. 
[44] Geert W Schmid-Schönbein, Shunichi Usami, Richard Skalak, and Shu Chien. The interaction of leukocytes and erythrocytes in capillary and postcapillary vessels. Microvasc. Res., 19(1):45-70, 1980.

[45] Harry L Goldsmith and Samira Spain. Margination of leukocytes in blood flow through small tubes. Microvasc. Res., 27(2):204-222, 1984.

[46] Wolfgang Weninger, Maté Biro, and Rohit Jain. Leukocyte migration in the interstitial space of non-lymphoid organs. Nat. Rev. Immunol., 14(4):232, 2014 .

[47] Peter Friedl and Katarina Wolf. Proteolytic interstitial cell migration: a five-step process. Cancer Metast. Rev., 28(1-2):129-135, 2009.

[48] Janine L Coombes and Ellen A Robey. Dynamic imaging of host-pathogen interactions in vivo. Nat. Rev. Immunol., 10(5):353, 2010.

[49] Chun-Min Lo, Hong-Bei Wang, Micah Dembo, and Yu-li Wang. Cell movement is guided by the rigidity of the substrate. Biophys. J., 79(1):144-152, 2000 .

[50] Robert J Pelham and Yu-li Wang. Cell locomotion and focal adhesions are regulated by substrate flexibility. Proc. Natl. Acad. Sci., 94(25):13661$13665,1997$.

[51] Shelly R Peyton and Andrew J Putnam. Extracellular matrix rigidity governs smooth muscle cell motility in a biphasic fashion. J. Cell. Physiol., 204(1):198-209, 2005.

[52] Theresa A Ulrich, Elena M de Juan Pardo, and Sanjay Kumar. The mechanical rigidity of the extracellular matrix regulates the structure, motility, and proliferation of glioma cells. Cancer Res., 69(10):4167-4174, 2009.

[53] Amit Pathak and Sanjay Kumar. Independent regulation of tumor cell migration by matrix stiffness and confinement. Proc. Natl. Acad. Sci., 109(26):10334-10339, 2012.

[54] Margareta M Mueller and Norbert E Fusenig. Friends or foesbipolar effects of the tumour stroma in cancer. Nature Rev. Cancer, 4(11):839, 2004.

[55] Matthew J Paszek, Nastaran Zahir, Kandice R Johnson, Johnathon N Lakins, Gabriela I Rozenberg, Amit Gefen, Cynthia A Reinhart-King, Susan S Margulies, Micah Dembo, David Boettiger, et al. Tensional homeostasis and the malignant phenotype. Cancer Cell, 8(3):241-254, 2005.

[56] Hong-Bei Wang, Micah Dembo, and Yu-Li Wang. Substrate flexibility regulates growth and apoptosis of normal but not transformed cells. Am. J. Physiol. Cell. Physiol., 279(5):C1345-C1350, 2000. 
[57] Paolo P Provenzano, David R Inman, Kevin W Eliceiri, Steven M Trier, and Patricia J Keely. Contact guidance mediated three-dimensional cell migration is regulated by rho/rock-dependent matrix reorganization. Biophys. J., 95(11):5374-5384, 2008.

[58] Rodrigo Ledesma-Aguilar and Julia M Yeomans. Enhanced motility of a microswimmer in rigid and elastic confinement. Phys. Rev. Lett., 111(13):138101, 2013.

[59] Lisa J Fauci and Amy McDonald. Sperm motility in the presence of boundaries. Bull. Math. Biol., 57(5):679-699, 1995.

[60] Vaseem A Shaik and Arezoo M Ardekani. Motion of a model swimmer near a weakly deforming interface. J. Fluid Mech., 824:42-73, 2017.

[61] Wolfgang Helfrich. Elastic properties of lipid bilayers: theory and possible experiments. Zeitschrift für Naturforschung C, 28(11-12):693-703, 1973.

[62] Badr Kaoui, GH Ristow, Isabelle Cantat, Chaouqi Misbah, and Walter Zimmermann. Lateral migration of a two-dimensional vesicle in unbounded poiseuille flow. Phys. Rev. E, 77(2):021903, 2008.

[63] AD Garrad and PW Carpenter. A theoretical investigation of flow-induced instabilities in compliant coatings. J. Sound Vib., 85(4):483-500, 1982.

[64] PW Carpenter and AD Garrad. The hydrodynamic stability of flow over kramer-type compliant surfaces. part 1. tollmien-schlichting instabilities. $J$. Fluid Mech., 155:465-510, 1985.

[65] PW Carpenter and AD Garrad. The hydrodynamic stability of flow over kramer-type compliant surfaces. part 2. flow-induced surface instabilities. J. Fluid Mech., 170:199-232, 1986.

[66] J Andrzej Domaradzki and Ralph W Metcalfe. Stabilization of laminar boundary layers by compliant membranes. Phys. Fluids, 30(3):695-705, 1987.

[67] Ralph W Metcalfe, Francois Battistoni, Johan Ekeroot, and Steven A Orszag. Evolution of boundary layer flow over a compliant wall during transition to turbulence. In Boundary Layer Transition and Control, pages $36-1,1991$.

[68] J Beaucourt, T Biben, and C Misbah. Optimal lift force on vesicles near a compressible substrate. EPL, 67(4):676, 2004.

[69] Constantine Pozrikidis. Boundary integral and singularity methods for linearized viscous flow. Cambridge University Press, 1992.

[70] Constantine Pozrikidis. Modeling and simulation of capsules and biological cells. CRC Press, 2003. 
[71] Shravan K Veerapaneni, Denis Gueyffier, Denis Zorin, and George Biros. A boundary integral method for simulating the dynamics of inextensible vesicles suspended in a viscous fluid in 2d. J. Comput. Phys., 228(7):2334$2353,2009$.

[72] Alexander Farutin, Thierry Biben, and Chaouqi Misbah. 3d numerical simulations of vesicle and inextensible capsule dynamics. J. Comput. Phys., 275:539-568, 2014.

[73] Itsushi Minoura and Ritsu Kamiya. Strikingly different propulsive forces generated by different dynein-deficient mutants in viscous media. Cell Motil. Cytoskeleton, 31(2):130-139, 1995.

[74] Toshiki Yagi, Itsushi Minoura, Akiko Fujiwara, Ryo Saito, Takuo Yasunaga, Masafumi Hirono, and Ritsu Kamiya. An axonemal dynein particularly important for flagellar movement at high viscosity implications from a new chlamydomonas mutant deficient in the dynein heavy chain gene dhc9. $J$. Biol. Chem., 280(50):41412-41420, 2005.

[75] Elizabeth H Harris. The Chlamydomonas Sourcebook: Introduction to Chlamydomonas and Its Laboratory Use, volume 1. Academic Press, 2009.

[76] Knut Drescher, Jörn Dunkel, Luis H Cisneros, Sujoy Ganguly, and Raymond E Goldstein. Fluid dynamics and noise in bacterial cell-cell and cell-surface scattering. Proc. Natl. Acad. Sci., 108(27):10940-10945, 2011.

[77] Eric Lauga and Thomas R Powers. The hydrodynamics of swimming microorganisms. Rep. Prog. Phys, 72(9):096601, 2009.

[78] Darci T Butcher, Tamara Alliston, and Valerie M Weaver. A tense situation: forcing tumour progression. Nature Rev. Cancer, 9(2):108, 2009.

[79] Ilya Levental, Penelope C Georges, and Paul A Janmey. Soft biological materials and their impact on cell function. Soft Matter, 3(3):299-306, 2007.

[80] Thomas R Cox and Janine T Erler. Remodeling and homeostasis of the extracellular matrix: implications for fibrotic diseases and cancer. Dis. Models Mech., 4(2):165-178, 2011.

[81] BU Felderhof. Swimming at low reynolds number of a cylindrical body in a circular tube. Phys. Fluids, 22(11):113604, 2010.

[82] Lailai Zhu, Eric Lauga, and Luca Brandt. Low-reynolds-number swimming in a capillary tube. J. Fluid Mech., 726:285-311, 2013.

[83] Bin Liu, Kenneth S Breuer, and Thomas R Powers. Propulsion by a helical flagellum in a capillary tube. Phys. Fluids, 26(1):011701, 2014. 
[84] Alejandro Bilbao, Eligiusz Wajnryb, Siva A Vanapalli, and Jerzy Blawzdziewicz. Nematode locomotion in unconfined and confined fluids. Phys. Fluids, 25(8):081902, 2013.

[85] Saikat Jana, Soong Ho Um, and Sunghwan Jung. Paramecium swimming in capillary tube. Phys. Fluids, 24(4):041901, 2012.

[86] Sir James Lighthill. Mathematical biofluiddynamics. SIAM, 1975.

[87] Edward M Purcell. The efficiency of propulsion by a rotating flagellum. Proc. Natl. Acad. Sci., 94(21):11307-11311, 1997.

[88] Leif E Becker, Stephan A Koehler, and Howard A Stone. On self-propulsion of micro-machines at low reynolds number: Purcell's three-link swimmer. J. Fluid Mech., 490:15-35, 2003.

[89] A Farutin, H Wu, W-F Hu, S Rafaï, P Peyla, M-C Lai, and C Misbah. Analytical study for swimmers in a channel. J. Fluid Mech., 881:365-383, 2019 .

[90] Madhav Ranganathan, Alexander Farutin, and Chaouqi Misbah. Effect of cytoskeleton elasticity on amoeboid swimming. Biophysical journal, 115(7):1316-1329, 2018.

[91] Alexander Farutin, Jocelyn Étienne, Chaouqi Misbah, and Pierre Recho. Crawling in a fluid. Phys. Rev. Lett., 123:118101, Sep 2019. 


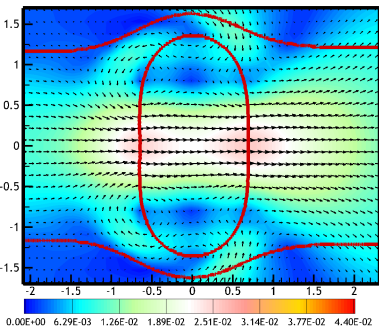

(a)

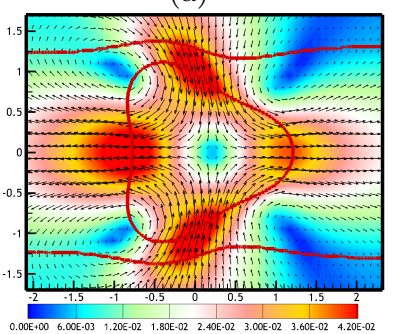

(b)

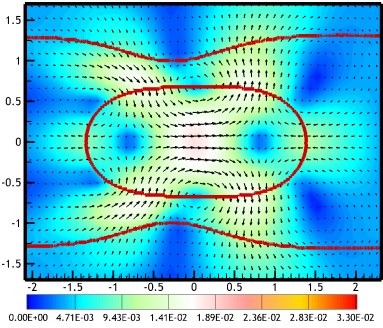

(c)

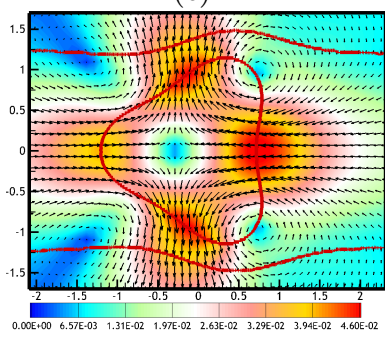

(d)

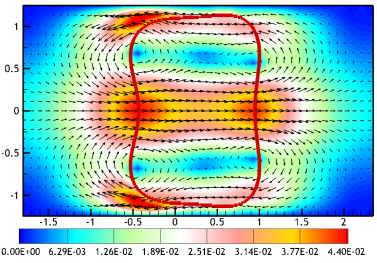

(e)

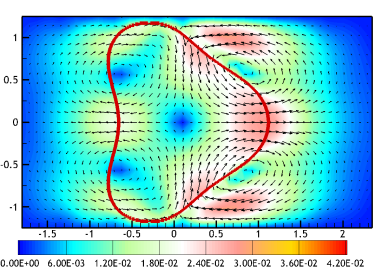

(f)

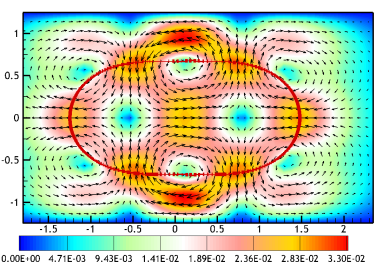

(g)

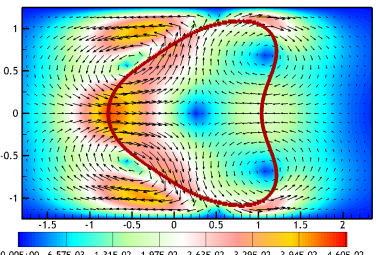

(h)

Figure 5: (color online) Comparison of velocity vector field superimposed over contours of velocity magnitude for an axially moving amoeboid swimmer in the flexible $\left(K_{w}=10\right)$ (left column) and rigid $\left(K_{w}=\infty\right)$ (right column) channels of confinement $C=0.8$ during four shape changes, namely, oblate in subfigures (a) and (e), pusher in subfigures (b) and (f), prolate in subfigures (c) and (g) and puller in subfigures (d) and (h) over one swimming stroke. Here subfigures in left and right columns correspond to flexible $\left(K_{w}=10\right)$ and rigid $\left(K_{w}=\infty\right)$ channels respectively. 


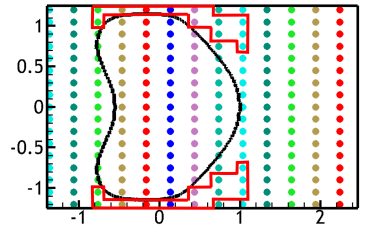

(a)

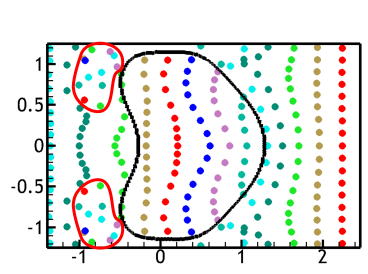

(b)

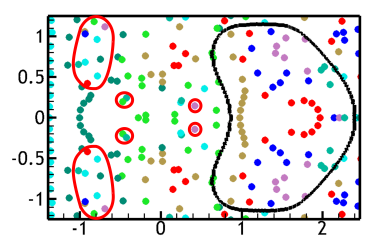

(c)

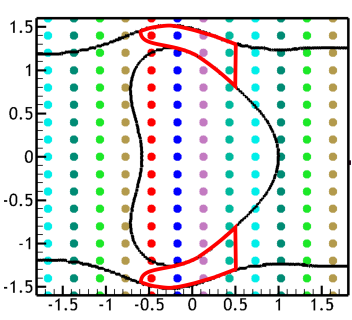

(d)

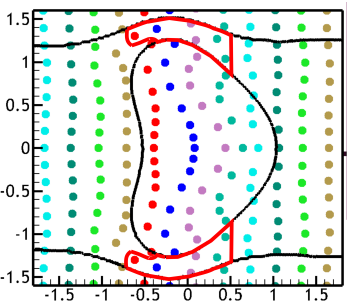

(e)

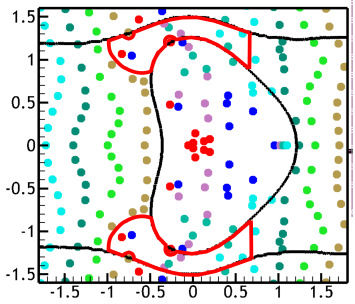

(f)

Figure 6: (color online) Comparison of snapshots of positions of massless tracer particles that follow flow field of an amoeboid swimmer suspended in the rigid $\left(K_{w}=\infty\right)$ (left column) and flexible $\left(K_{w}=10\right)$ (right column) channels of confinement $C=0.8$ at time $t=t_{o}$ (some initial time) in subfigures (a) and (d),$t=t_{o}+T_{s}$ (after one swimming stroke) in subfigures (b) and (e) and $t=t_{o}+5 \times T_{s}$ (after five swimming strokes) in subfigures (c) and (f). Here subfigures in left and right column correspond to the rigid $\left(K_{w}=\infty\right)$ and flexible $\left(K_{w}=10\right)$ channel respectively. 


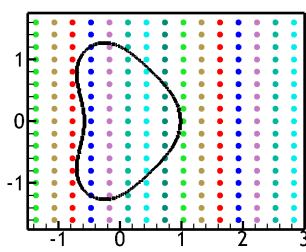

(a)

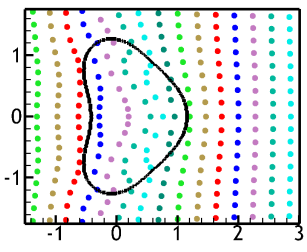

(b)

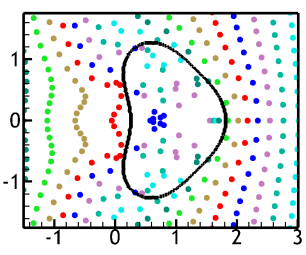

(c)

Figure 7: (color online) Snapshots of positions of massless tracer particles that follow flow field of an amoeboid swimmer suspended in an unconfined fluid at time, $t=t_{o}$ (some initial time) in subfigure (a), $t=t_{o}+T_{s}$ (after one swimming stroke) in subfigure (b) and $t=t_{o}+5 \times T_{s}$ (after five swimming strokes) in subfigure (c).

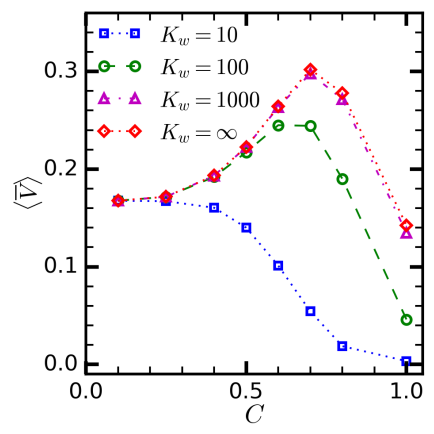

Figure 8: (color online) Variation of time averaged velocity, $\langle\bar{V}\rangle=$ $\left(\bar{X}_{c}\left(t+T_{s}\right)-\bar{X}_{c}(t)\right)$, over swimming stroke with confinement, $C$, for an axially moving amoeboid swimmer in flexible channels of wall stiffnesses, $K_{w}=10,100$, 1000 and $\infty$. The dimensionless quantities are $\bar{V}=V T_{c} / R_{o}$ and $\bar{X}_{c}=X_{c} / R_{o}$. 


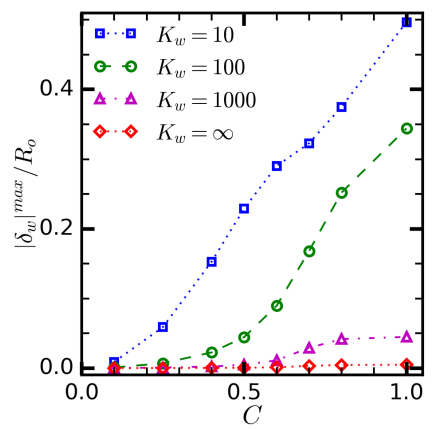

(a)

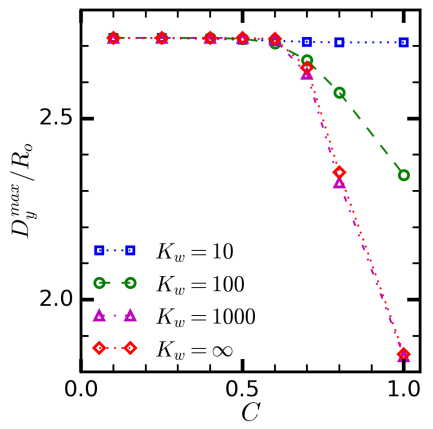

(b)

Figure 9: (color online) Variation of (a) maximum wall deformation, $\left|\delta_{w}\right|^{\text {max }}$, and (b) maximum lateral elongation, $D_{y}^{\max }$, of the swimmer over swimming stroke with confinement, $C$, for an axially moving amoeboid swimmer in flexible channels of wall stiffnesses $K_{w}=10,100,1000$ and $\infty$. 


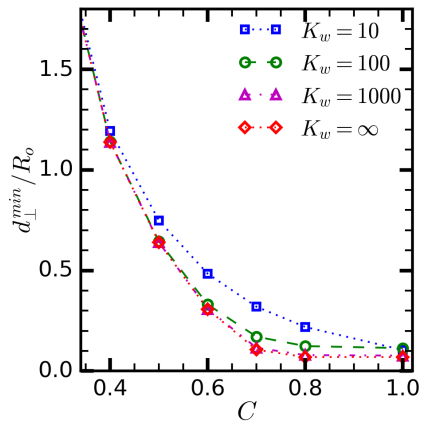

(a)

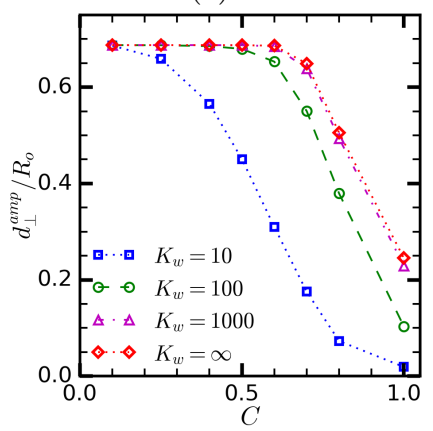

(b)

Figure 10: (color online) Variation of (a) minimum film thickness, $d_{\perp}^{\text {min }}$, and (b) amplitude of variation in film thickness, $d_{\perp}^{a m p}=d_{\perp}^{\text {max }}-d_{\perp}^{\text {min }}$, between swimmer and channel walls, over swimming stroke with confinement, $C$, for an axially moving amoeboid swimmer in flexible channels of wall stiffnesses $K_{w}=10,100$, 1000 and $\infty$. 


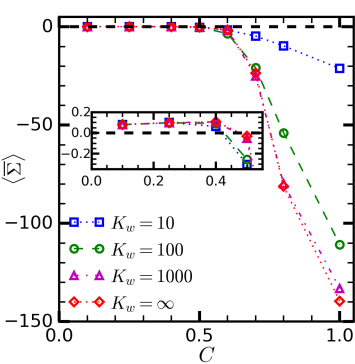

(a)

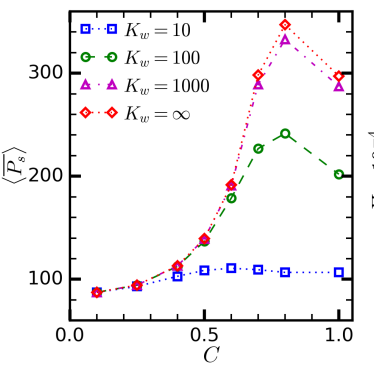

(b)

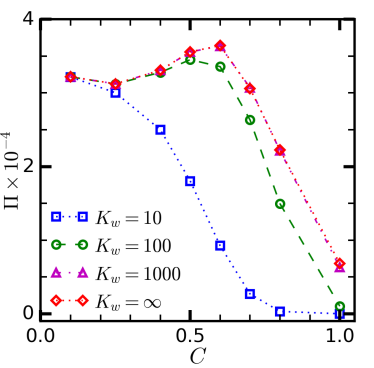

(c)

Figure 11: (color online) Variation of (a) time averaged stresslet, $\langle\bar{\Sigma}\rangle=$ $\left(\int_{0}^{T_{s}} \bar{\Sigma} d t\right) / T_{s}$, where $\bar{\Sigma}=\left(\sigma_{x x}-\sigma_{y y}\right) /\left(\eta R_{o}^{2} / T_{s}\right), \sigma_{i j}=\oint_{\Omega_{3}} f_{i} r_{j} d s$, (b) time averaged power consumption, $\left\langle\bar{P}_{s}\right\rangle=\left\langle P_{s}\right\rangle T_{s}^{2} /\left(\eta R_{o}^{2}\right)$ where $\left\langle P_{s}\right\rangle=$ $\frac{1}{T_{s}} \int_{0}^{T_{s}} \oint_{\Omega_{3}}(\mathbf{f}(s) \cdot \mathbf{u}(s)) d s d t=\left\langle\oint_{\Omega_{3}}(\mathbf{f}(s) \cdot \mathbf{u}(s)) d s\right\rangle$, and (c) efficiency of the swimmer, $\Pi=\eta\langle\bar{V}\rangle^{2} /\left\langle\overline{P_{s}}\right\rangle$, over swimming stroke with confinement, $C$, for an axially moving amoeboid swimmer in flexible channels of wall stiffnesses $K_{w}=$ $10,100,1000$ and $\infty$. 